\title{
Laryngofissure in comorbid patient under ultrasound-guided regional anesthesia of the neck
}

\author{
Bahaa Mohammed Refaie ${ }^{1 *}$ (D) Mohammed Elrabie Ahmed ${ }^{2}$, Mohammed Ahmed Ibrahim ${ }^{1}$ and \\ Farghali Abdelrahman²
}

\begin{abstract}
Background: Major neck surgery is done typically under general anesthesia (GA). The neural blocks of the neck include blocking of the cervical plexus, superior laryngeal nerve, trans-laryngeal, block of the glossopharyngeal nerve, and local anesthetic infiltration. In patients with a high risk of GA, including those with pulmonary dysfunction, and prior myocardial ischemia or infarction, regional anesthesia is mainly indicated.

Case presentation: We record a case of a comorbid geriatric patient with dysphonia and left glottic mass that was diagnosed as squamous cell papilloma by transoral biopsy using curved biopsy forceps under local spray anesthesia, and after 6 months, this patient developed stridor for which tracheostomy, laryngofissure, and left cordectomy were then performed solely under neck blocks. Surgery was performed while the patient remained pain-free and stable without any morbidity throughout the operation.
\end{abstract}

Conclusions: In high-risk patients and low-resource health systems, regional anesthesia in neck surgery can be a reasonable and cheap alternate to general anesthetics.

Keywords: Regional, Cervical, Neck, Block, Laryngeal

\section{Background}

General anesthesia (GA) is widely applied in neck operations, but patients with head and neck cancer and some other elderly patients often suffer from COPD, coronary atherosclerosis, and other comorbidities which together with the risks of arrhythmogenic inhalation anesthetics and assisted ventilation during general anesthesia make them at high risk of general anesthesia up to be considered as non-operable cases. Any preoperative attempts to enhance these patients' cardiopulmonary reserves as respiratory therapy with or without bronchodilators seldom alter the clinical risk [1]. Regional anesthesia (RA) is used less frequently in the head and neck. The cervical plexus blocks (CPBs) can provide efficient head and neck

\footnotetext{
* Correspondence: bahaarefaay@med.sohag.edu.eg

'Anesthesia and ICU Department, Faculty of Medicine, Sohag University, Sohag, Egypt

Full list of author information is available at the end of the article
}

anesthesia and analgesia [2]; carotid endarterectomy (CEA) and analgesia after neck operations have been the most common clinical applications for CPBs [3]; cervical plexus block may be enough for thyroidectomy, but the utilization of RA remains controversial in this type of operations [2]. Since the use of ultrasonography in the head and neck area has increased, CPBs can be done more precisely under the guidance of ultrasonography, which facilitates the identification of several vital landmarks including the muscles, vertebrae, main vessels, nerves, and fascia [4]. This paper aims to explain the procedure, indications, and limits of using neck RA alone in highly comorbid patients as a safe alternative to GA, we present a case of huge left glottic papilloma presented with dysphonia and stridor with extreme comorbidities in which tracheostomy then laryngofissure and cordectomy were done under a regional block. This is the first report of cordectomy through laryngofissure done solely under the regional neck block. 


\section{Case presentation}

A 71-year-old male patient with a history of diabetes, hypertension, COPD, and cardiac (echocardiography showing global hypokinesia with ejection fraction of 35\%) and chronic kidney diseases (elevated creatinine $3.5 \mathrm{mg} / \mathrm{dl}$ ) presented to our Otolaryngology department with dysphonia 1 year ago without difficulty in swallowing or breathing. Neck examination was free. Naso-fiberoptic examination revealed a whitish exophytic left glottic mass with restricted mobility (Fig. 1a). A computer tomography scan was done and showed a mass affecting the right glottic cord without cartilage invasion. As regarding the ASA (American Society of Anesthesiologists), the patient was ASA IV with high risk for general anesthesia. Transoral biopsy under local spray anesthesia was done using curved biopsy forceps guided by naso-fiberoptic laryngoscopy which revealed squamous cell papilloma. Six months later, the patient has developed mild to moderate stridor. CT neck showed a huge mass arising from the left vocal cord filling the glottis and subglottic area without cartilage invasion (Fig. 2). After a discussion with our anesthesia team, surgery was planned to be done under regional anesthesia to avoid the risks of general anesthesia.

We decided to do tracheostomy under a bilateral cervical block, bilateral superior laryngeal block, translaryngeal, and local infiltration anesthesia. Then, a laryngofissure approach was performed by splitting the thyroid cartilage, and left cordectomy was done. The entire mass was removed in piecemeal and sent for histopathological examination. The biopsy was early squamous cell carcinoma on top of squamous cell papilloma. The patient was T2NOM0. He refused to undergo any further surgery and accepted radiotherapy (60 Gy on 35 cycles). Tracheostomy weaning was done successfully 3 months later with a good and adequate respiratory gap. One-year follow-up is good with no evidence of residual lesions (Figs. 1b and Fig. 3).

\section{Anesthesia technique}

After intensive patient counseling about his morbidities and the risks of general anesthesia and getting informed written consent from him about the plan of anesthesia and the surgery and checking normal coagulation profile, the procedure was done using bilateral superficial cervical plexus block under ultrasound guidance where the patient was in the dorsal position, with his cranium tilted to either side for the bilateral block. The ultrasound screen was contralateral to the clinician, and the ultrasound transducer directional marker was medial. Under complete aseptic conditions, a high-frequency linear transducer was placed transversely on the anterior neck at the middle of the line connecting the mastoid process with the insertion of the sternal head of sternocleidomastoid muscle (SCM).

The target is to guide the tip of the needle medially just below the tapering posterolateral end of the SCM to the fascial plane below the SCM and just over the levator scapulae. An out-of-plane approach was applied. After aspiration, 1-2 $\mathrm{mL}$ of local anesthetic was injected to verify the appropriate injection position. The rest of the local anesthetic ( $8 \mathrm{~mL}$ on each side) was administered to envelop the plexus. The superior laryngeal block was also done to anesthetize the larynx from the epiglottic area to the cords where the hyoid is displaced towards the side to be blocked by grasping the hyoid between the index and the thumb, and the syringe is then walked off the caudal edge of the greater hyoid cornu and pushed 2 to $3 \mathrm{~mm}$ so that the tip becomes between the thyrohyoid membrane and the laryngeal mucosa; $2-3 \mathrm{~mL}$ of local anesthetic is then placed. Before opening the trachea in tracheostomy, trans-laryngeal block to anesthetize the subglottic region and the trachea was done where the patient is asked to exhale forcefully, and then $3 \mathrm{~mL}$ of $2 \%$ lignocaine is injected through the cricothyroid membrane while the patient takes inspiration.

The procedure duration was about $2.5 \mathrm{~h}$ during which the patient was calm on delivering supplemental oxygen at a flow

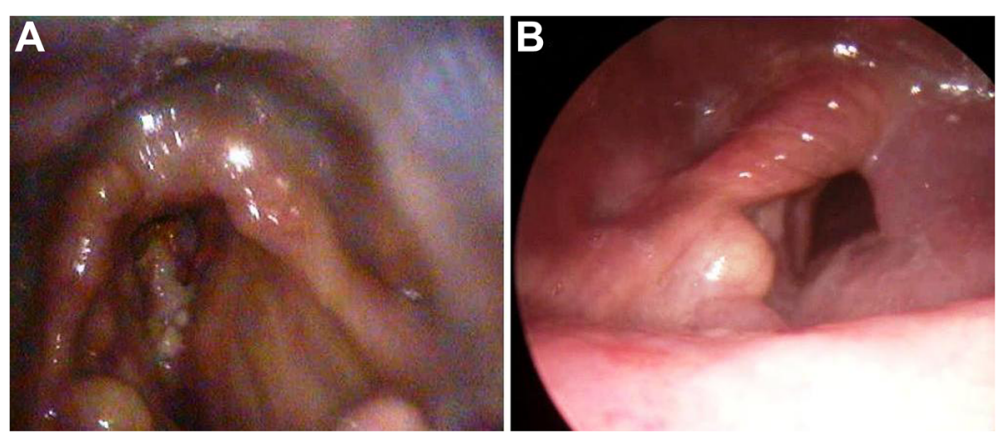

Fig. 1 Preoperative naso-fiberoptic laryngeal examination (a). Postoperative naso-fiberoptic laryngeal examination (b) 


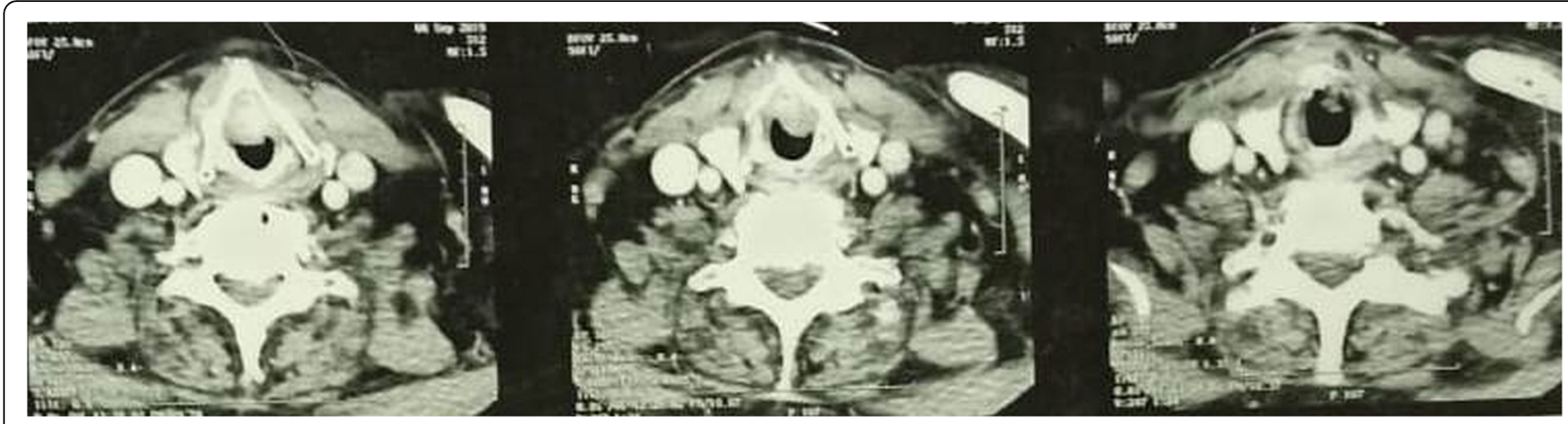

Fig. 2 Preoperative CT neck

of $3 \mathrm{l} / \mathrm{min}$ through tracheostomy with no need for adjuvant anesthesia or analgesia; as surgery ends, the patient is discharged to the recovery room for follow-up. The postoperative period was uneventful with no nausea or vomiting, and the need for postoperative analgesia was approximately after $6 \mathrm{~h}$ following the surgery which was achieved with a combination of ketorolac and paracetamol.

\section{Discussion}

Neck surgeries are usually done under general anesthesia (GA). However, there is still a shortage of anesthetists, anesthetic medications, and equipment in the developing countries, and many patients have multiple comorbidities which make regional neck anesthesia (RA) a safe and effective alternative to GA with non-significant complication [5].

Neck RA was usually used as an additive to GA or combined with sedation to lighten the depth of GA, provide sustained postoperative analgesia, and decrease RA requirements [5-8]. But, the recent papers and the conclusions from the literature on neurosurgery and vascular surgeries indicate that regional anesthesia alone may be associated with a better prognosis in these operations, especially endarterectomy of the carotid [5].

The GALA study (General Anesthetic versus Local Anesthetic) examined the assumption that RA in carotid endarterectomy is more safe than GA in a big number of patients [5]. Most of the local anesthetic strategies are fairly low-risk procedures including the superficial cervical plexus block $[5,8]$.

The improvement in the use of US guidelines in the RA provided a stronger and accurate outcome where the precise position of the cervical plexus can be found, up to visualize the nerve roots to be blocked $[9,10]$.

The cervical block is a prevalent RA technique developed in the early 20th century. Heidenhein, 1914, identified the lateral approach to the cervical block which presented the base for cervical plexus anesthetic techniques $[7,11]$. The block is done either in the deep or superficial plane or both.

Superficial and deep cervical blocks can provide anesthesia of several surgeries including superficial neck surgery (as lymph node dissection), thyroidectomy, carotid endarterectomy, in which awake neurological observation represents an effective neurological evaluation method [8].

There are no strict recommendations for carrying out large neck operations under RA, but, RA may be optimal in those with a high risk of GA, pulmonary dysfunction, prior cardiac infarctions, patients needing urgent laryngectomy, those preferring local to general anesthesia, and patients whose surgeons favor RA. Hochmann and

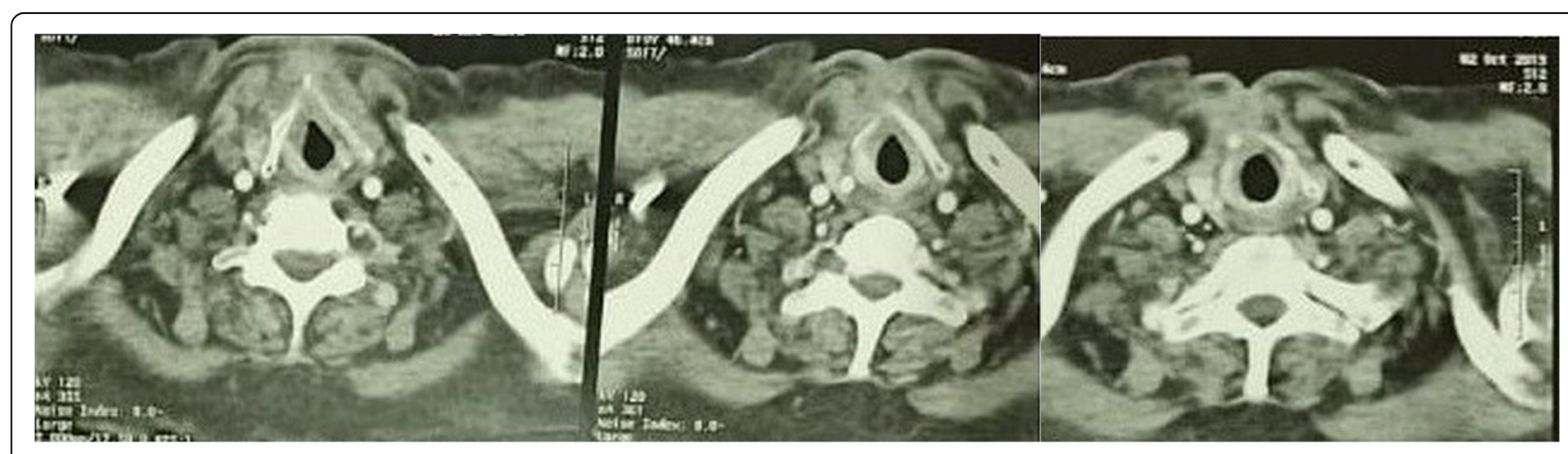

Fig. 3 Postoperative $C T$ neck 
Willard both thought that a positive family or personal history of malignant hyperthermia may be an indication of surgery under local anesthesia [10].

RA bypasses the risks associated with GA (e.g., endotracheal intubation complications and anesthetic agent unpleasant effects). RA also enables intraoperative voice monitoring and offers excellent postoperative analgesia [12], although there are still some drawbacks to RA techniques, including the possibility of local anesthetic toxicity, spinal cord, or epidural injections, neuropraxia, and hematoma formation in anticoagulant patients in particular $[11,13]$. The blockage of the phrenic nerve does not happen with the superficial cervical block; however, it is frequent with the deep plexus block [7]. Bilateral deep block can cause extreme breathing impairment due to paralysis of the diaphragm and is thus not advised. The central nervous system toxicity is the most significant complication of the cervical plexus block. This complication is due to the high vascularity of the neck, including both the vertebral and the carotid arteries, and is typically triggered by unintentional local anesthetic injection intravascularly more than absorption [5-7]. Cautious aspiration while the local anesthetic is being administered is a must. In an awake patient, surgical operation around the neck can cause anxiety, fear, and panic feelings. Preoperative psychotherapy and reassurance are of vital importance for alleviating patient anxiety.

Mausumi, 2012, enrolled 136 cases of neck operations including excision of the thyroglossal cyst, thyroglossal fistula or branchial fistula, biopsy or excision of the lymph node, thyroidectomy, and other neck swellings, including sebaceous cyst, lipoma, and granuloma in uncontrolled prospective research in which all candidates received ropivacaine $0.5 \%$ for a bilateral cervical plexus block. Lignocaine $1 \%$ and adrenaline $1: 100,000$ were used for infiltrating the incision line. For thyroglossal fistula and thyroglossal cyst, a further $1.5 \mathrm{~mL}$ of LA solution was injected on both sides of the midline over the hyoid bone. Inadequate anesthesia was managed by using $25 \mathrm{mg}$ ketamine IV which was repeated when necessary, and he confirmed that the net success of the bilateral cervical plexus block as a single anesthetic procedure in these neck operations was about $92 \%$ and that it exceeded more than $99 \%$ with supplementation of low-dose ketamine. For this study, however, thyroidectomy was not successful under the cervical plexus block [8].

Kishore C., 1998, reported 50 patients enrolled in major neck surgery (ten laryngectomies with or without neck dissection, thirty thyroidectomies, two branchial cysts, five submandibular gland, and three thyroglossal cyst excisions) under RA and found that combining regional blocks and intraoperative sedatives provided excellent patient satisfaction and analgesia. The blood loss intraoperatively was small, limited complications after surgery, and quicker recovery, but for many reasons, RA was less successful for surgical procedures extending more than $4 \mathrm{~h}$. Firstly, lignocaine's anesthetic effect, though mixed with adrenaline, is wearing off. Secondly, the patient becomes agitated from staying for extended periods in one position. In the end, the cervical block does not anesthetize the posterior triangle of the neck adequately and is, therefore, less efficient for performing neck dissection [10].

Here, our paper presents a case of left glottic mass associated with stridor in comorbid patients where tracheostomy, laryngofissure, and left cordectomy were performed under neck RA solely to prevent the high risk of GA. We used bupivacaine $0.5 \%$ to provide appropriate anesthesia time, and the operation lasted for around $2 \mathrm{~h}$ without any intra or postoperative problems. The patient was calm intraoperatively and had strong postoperative analgesia with early recovery.

\section{Conclusion}

The regional anesthesia of the neck alone is well tolerated and is of great value as it requires the least resources. Laryngofissure or laryngectomy can be safely performed especially in cases with multiple comorbidities with excellent postoperative analgesia, patient satisfaction, and early recovery and ambulation. This positive experience from this case will encourage us to continue using regional anesthesia with monitored anesthesia care in high-risk patients in other neck operations.

\section{Abbreviations \\ ASA: American Society of Anesthesiologists; GA: General anesthesia; \\ CEA: Carotid endarterectomy; COPD: Chronic obstructive pulmonary disease; \\ CPB: Cervical plexus block; CT: Computed tomography; DM: Diabetes \\ mellitus; LA: Local anesthetic; RA: Regional anesthesia; \\ SCM: Sternocleidomastoid muscle; US: Ultrasonography}

\section{Acknowledgements \\ None}

\section{Authors' contributions}

All authors participated in manuscript processing and revision. All authors have read and approved the final manuscript for submission. BR was responsible for the review of literature, drafting the manuscript, and review and approval of the final copy of the manuscript. MA did the surgery and video documentation, follow-up of the patient, and review and approval of the final copy of the manuscript. MI was responsible for the anesthesia and video documentation and review and approval of the final copy of the manuscript. FA did the collection of data and follow-up and review and approval of the final copy of the manuscript.

\section{Funding}

None

Availability of data and materials

Any data or materials related to the case are readily available for revision.

Ethics approval and consent to participate

This report complies with regional and institutional ethical guidelines and with declaration of Helsinki. A written informed consent was obtained from our case for her participation. 


\section{Consent for publication}

A written informed consent to publish the clinical data was obtained from our patient involved in this case report.

\section{Competing interests}

None

\section{Author details}

'Anesthesia and ICU Department, Faculty of Medicine, Sohag University, Sohag, Egypt. ${ }^{2}$ ENT Department, Faculty of Medicine, Sohag University, Sohag, Egypt.

Received: 22 April 2020 Accepted: 5 February 2021

Published online: 03 March 2021

\section{References}

1. Wittch DJ JJ, Berny JJ, Davis RK (1984) Cervical epidural anesthesia for head and neck surgery. Laryngoscope. 94:615-619

2. Andrieu G, Amrouni $H$, Robin E et al (2007) Analgesic efficacy of bilateral superficial cervical plexus block administered before thyroid surgery under general anesthesia. Br J Anaesth. 99:561-566

3. Ivanec Z, Mazul-Sunkol B, Lovricević I et al (2008) Superficial versus combined (deep and superficial) cervical plexus block for carotid endarterectomy. Acta Clin Croat. 47:81-86

4. Saranteas T, Paraskeuopoulos T, Anagnostopoulou S et al (2010) Ultrasound anatomy of the cervical paravertebral space: a preliminary study. Surg Radiol Anat. 32:617-622

5. Salwa Mohamed SH, El-B HM, Eiad AR et al (2012) Efficacy of unilateral combined (superficial and deep) cervical plexus block as preemptive analgesia for unilateral neck dissection surgery. Egypt J Anaesthesia. 28(4): 275-279

6. Ducic Y, Young L, McIntyre J (2010) Neck dissection past and present. Minerva Chirurgica. 65(1):45-58

7. Roger DM, Elizabeth JC, Manuel RC et al (1995) Superficial and deep cervical plexus block: technical considerations. J Am Assoc Nurse Anesthetists. 63(3): 235-243

8. Mukhopadhyay S, Niyogi M, Dutta M, Ray R et al (2012) Bilateral superficial cervical plexus block with or without low-dose intravenous ketamine analgesia: effective, simple, safe, and cheap alternative to conventional general anesthesia for selected neck surgeries. Local Reg Anesth. 5:1-7

9. Shanthanna H (2014) Ultrasound-guided selective cervical nerve root block and superficial cervical plexus block for surgeries on the clavicle. Indian J Anaesth. 58(3):327-329

10. Prasad KC, Shanmugam VU (1998) Major neck surgeries under regional anesthesia. Am J Otolaryngol. 19(3):163-169

11. www.nysora.com/techniques/head-and-neck-blocks/cervical/ultrasoundguided-cervical-plexus-block/

12. Lee $\mathrm{JH}$, Yoo JH, Cho SH et al (2009) Thyroid surgery under monitored anesthesia care (MAC). Korean J Anaesthesiol 56:284

13. Carling A, Simmonds M (2000) Complications from regional anesthesia for carotid endarterectomy. Br J Anaesth 84:797-800

\section{Publisher's Note}

Springer Nature remains neutral with regard to jurisdictional claims in published maps and institutional affiliations.

\section{Submit your manuscript to a SpringerOpen ${ }^{\circ}$ journal and benefit from:}

- Convenient online submission

- Rigorous peer review

- Open access: articles freely available online

- High visibility within the field

- Retaining the copyright to your article

Submit your next manuscript at $\boldsymbol{\nabla}$ springeropen.com 